\title{
Investigating Overdensities around $z>6$ Galaxies through ALMA Observations of [C II]
}

\author{
Tim B. Miller ${ }^{1,2}$, Scott C. Chapman ${ }^{1,3}$, Christopher C. Hayward ${ }^{4}$ (ㅁ) Peter S. Behroozi ${ }^{5}$, Matt Bradford ${ }^{6,7}$, Chris J. Willott ${ }^{8}$ (i), and \\ Jeff Wagg 9 \\ ${ }^{1}$ Department of Physics and Atmospheric Science, Dalhousie University, 6310 Coburg Road, Halifax, NS B3H 4R2, Canada; tim.miller@yale.edu \\ ${ }^{2}$ Department of Astronomy, Yale University, 52 Hillhouse Avenue, New Haven, CT 06511, USA \\ ${ }^{3}$ NRC Herzeberg, 5071 West Saanich Road, Victoria, BC V9E 2E7, Canada \\ ${ }^{4}$ Center for Computational Astrophysics, Flatiron Institute, 162 Fifth Avenue, New York, NY 10010, USA \\ ${ }^{5}$ Department of Astronomy and Steward Observatory, University of Arizona, Tucson, AZ 85721, USA \\ ${ }^{6}$ California Institute of Technology, 1200 East California Boulevard, Pasadena, CA 91125, USA \\ 7 Jet Propulsion Laboratory, 4800 Oak Grove Drive, Pasadena, CA 91109, USA \\ ${ }^{8}$ NRC Herzeberg, 5071 West Saanich Road, Victoria, BC V9E 2E7, Canada \\ ${ }^{9}$ Square Kilometre Array Organization, Jodrell Bank Observatory, Lower Withington, Macclesfield, Cheshire SK11 9DL, UK \\ Received 2018 September 19; revised 2019 December 16; accepted 2019 December 16; published 2020 January 29
}

\begin{abstract}
We present a search for companion [C II] emitters to known luminous sources at $6<z<6.5$ in deep, archival ALMA observations. The observations are deep enough to detect sources with $L_{[\mathrm{C} \text { II] }} \sim 10^{8} L_{\odot}$ at $z \sim 6$. We identify three new robust line detections from a blind search of five deep fields centered on ultraluminous infrared galaxies and QSOs. We calculate the volume density of companions and find a relative overdensity of $6_{-3}^{+4}$ and $86_{-37}^{+60}$ when comparing to current observational constraints and theoretical predictions, respectively. These results suggest that the central sources may be highly biased tracers of mass in the early universe. We find these companion lines to have comparable properties to other known galaxies at the same epoch. All companions lie less than $650 \mathrm{~km} \mathrm{~s}^{-1}$ and between 25 and $60 \mathrm{kpc}$ (projected) from their central source. To place these discoveries in context, we employ a mock galaxy catalog to estimate the luminosity function for [C II] during reionization and compare to our observations. The simulations support this result by showing a similar level of elevated counts found around such luminous [C II] sources.
\end{abstract}

Unified Astronomy Thesaurus concepts: Quasar-galaxy pairs (1316); Reionization (1383); Submillimeter astronomy (1647); High-redshift galaxies (734); Galaxies (573)

\section{Introduction}

In order to further our understanding of galaxy formation, we must investigate how the first galaxies formed during the epoch of reionization (EoR). Advances in (sub)millimeter interferometers have made it possible to detect galaxies out to a redshift of 6 and beyond both in continuum and spectroscopically (Riechers et al. 2013; Maiolino et al. 2015; Strandet et al. 2017; Marrone et al. 2018). This enables constraints on physical properties such as star formation rate (SFR), dynamical mass, and conditions in the interstellar medium (ISM; Wang et al. 2013; Willott et al. 2015a, 2015b). The most luminous galaxies and quasars found at $z>6$ are expected to be highly biased tracers of the underlying dark matter distribution, forming in the most overdense regions of space. Hierarchical evolution causes these overdensities to grow with time, making it likely that these systems are progenitors of the most massive galaxies and structures we observe at any redshift (Chiang et al. 2013). This makes observations of galaxies during the EoR a crucial probe of the early evolution of these massive systems. Observations of these extreme systems and their surroundings during the EoR are key to constraining galaxy formation models.

Given the importance of understanding and characterizing overdensities in the EoR, many studies have searched for overdensities the fields surrounding quasars as possible beacons of massive halos using various observational techniques. Early attempts leveraged the Lyman break technique to detect Lyman break galaxies (LBGs) by searching for dropouts in photometric data. This technique has yielded mixed results, with some studies reporting an excess of galaxies in the fields of quasars (Stiavelli et al. 2005; Zheng et al. 2006; Kim et al. 2009; Husband et al. 2013), others reporting no overdensity of galaxies (Willott et al. 2005; Utsumi et al. 2010), and Kim et al. (2009) even reported an underdensity of LBGs in two of the five quasar fields searched. A complimentary technique is to search for Ly $\alpha$ emitters (LAEs), which provides the advantage of searching a narrow redshift range $(\Delta z \sim 0.1)$ compared to the Lyman break technique $(\Delta z \sim 1)$ that may select galaxies that are physically unassociated with the quasar. This technique has produced similarly mixed results, with several studies reporting no detections of LAEs in the fields of quasars at $z>6$ (Bañados et al. 2013; Mazzucchelli et al. 2017; Goto et al. 2017), but Farina et al. (2017) reported the detection of an LAE in close vicinity to a $z \sim 6.6$ QSO. Combining these two techniques, Ota et al. (2018) investigated the environment of a quasar at $z=6.61$, searching for both LAEs and LBGs. They found an overdensity of LBGs but an underdensity of LAEs. It is likely that these techniques are probing different populations of galaxies, and the authors suggest that LAEs likely reside in lower-mass halos $\left(M_{\text {halo }} \lesssim 10^{10} M_{\odot}\right)$ than LBGs and thus are more easily quenched by the UV radiation field of the quasar. Champagne et al. (2018) searched for millimeter continuum sources in the fields surrounding 35 quasars at $z>6$ and found no evidence for an elevated number of sources in these fields. However, searching for galaxies using the (sub)millimeter technique only selects dusty and highly star-forming galaxies (SFR $\gtrsim 100 M_{\odot} \mathrm{yr}^{-1}$ ). Additionally, the negative $K$-correction of galaxies in this regime leads to a large line-of-sight volume 
probed, thereby potentially washing out any intrinsic overdensity.

Given these varied results, there are a few explanations for why quasars may not inhabit overdense regions in the early universe. Willott et al. (2005) suggested that, due to scatter in the $M_{\mathrm{BH}}$ versus $M_{\text {halo }}$ relation, quasars in the early universe may not populate as high-mass halos as previously expected. This implies that quasars would not actually signpost overdense regions in the early universe; therefore, finding companions would be less likely. Alternatively, Utsumi et al. (2010) and Mazzucchelli et al. (2017) suggested that the lack of companions may be due to strong UV radiation from the quasar inhibiting galaxy formation, even if they reside in massive halos. However, recent studies from McGreer et al. (2014) and Trakhtenbrot et al. (2017) have found galaxies within a projected $50 \mathrm{kpc}$ distance from quasars. Generally, quasars have not proved a reliable avenue to signpost overdensities at high redshifts, and previous studies seem to indicate a complex bias (e.g., Trainor \& Steidel 2012).

Attempts have also been made to search for galaxy overdensities around the most distant submillimeter galaxies (SMGs), most notably, HFLS3, which has a redshift of 6.34 (Riechers et al. 2013) and is one of the most extreme objects known to exist during the EoR. This object appears to be a massive starburst with an SFR of $\sim 2900 M_{\odot} \mathrm{yr}^{-1}$, with gas and dust masses of $1 \times 10^{11}$ and $1.3 \times 10^{9} M_{\odot}$, respectively. Following its discovery, two studies were conducted to search for an excess of galaxies in the surrounding field (Robson et al. 2014; Laporte et al. 2015). Robson et al. (2014) searched the field around HFLS3 with SCUBA2 at 450 and $850 \mu \mathrm{m}$ wavelengths. They found no evidence for an excess of luminous submillimeter emitters (with implied $L_{\mathrm{IR}}>5 \times 10^{12}$ ) on a scale of 1.5 Mpc around HFLS3. Laporte et al. (2015) used the Gran Telescopio Canarias (GTC) and the Hubble Space Telescope (HST) to search for an excess of LBGs in the same field. Even at the lower SFRs probed by the Lyman break technique, they did not find any significant evidence that HFLS3 is a member of a protocluster.

While these results appear somewhat at odds with expectations, the studies described above suffer due to the sensitivity limits and shortcomings of the selection techniques used. Optical selection of LBGs is difficult during the EoR due to the faintness of galaxies at $z>6$. Similarly, single-dish submillimeter observations only select galaxies with high SFRs $\left(\gtrsim 100 M_{\odot} \mathrm{yr}^{-1}\right)$ and will likely miss lower-mass galaxies detected through other methods. Even using more sensitive large interferometers, like the Atacama Large Millimeter/ submillimeter Array (ALMA), it has been shown that many UV selected galaxies are undetected in the far-IR (FIR) continuum (Bouwens et al. 2016). Using ALMA to instead observe ionized carbon emission lines is a complimentary method to detecting galaxies during the EoR. Carbon has one of the lowest ionization energies of the elements that are abundant in the early universe. Due to the fine structure of ionized carbon, [C II], it is excited at $91 \mathrm{~K}$ and then decays through the ${ }^{2} P_{3 / 2} \rightarrow{ }^{2} P_{1 / 2}$ transition, which emits a photon at $157.7 \mu \mathrm{m}$. One of the brightest emission lines in star-forming galaxies, $[\mathrm{C} \mathrm{II}]$ is a major cooling mechanism in the ISM. Recent works have shown that it is possible to study [C II] emission in high-redshift galaxies using ALMA. Capak et al. (2015) and Willott et al. (2015a) studied the FIR and dust properties of galaxies using ALMA, while many studies have probed the $[\mathrm{C}$ II] and dust of the host galaxies of $z \sim 6$ quasars (Wang et al. 2013; Willott et al. 2013; Decarli et al. 2018).

There are some downsides to using the [C II] emission line to search for companion galaxies. The procedure used to identify candidate sources by performing a blind search of 3D data cubes leads to many independent measurements that could produce a high rate of false positives (Aravena et al. 2016). Additionally, different emission lines originating from galaxies at lower redshifts can be confused with [C II] at $z \sim 6$. Specifically, the CO rotational lines corresponding to $J=3-5$ originating at $z \sim 0.5-2$ appear at the same observed frequency as $[\mathrm{C} \mathrm{II}]$ at $z \sim 6$. Decarli et al. (2017) searched for [C II]-emitting companions around 25 quasars at $z>6$. They found four companions at high significance $(>7 \sigma)$ with $L_{\text {[C II] }}>10^{9} L_{\odot}$. This is orders of magnitude more sources than expected given the volume probed by the ALMA observations. Therefore, the authors conclude that bright, high-redshift quasars provide beacons of dark matter overdensities in the early universe.

In this paper, we investigate the hypothesis that the environments of extreme objects at $z>6$ should possess overdensities of galaxies by performing a sensitive search for companions around quasars using [C II] emission lines. In Section 2 we define our sample ALMA fields and develop a method to search for robust [C II] line-emitting companions around previously observed extreme objects at $z \sim 6$. In Section 3 we describe the results of a similar analysis performed on a simulated galaxy sample from the Hayward et al. (2013a) mock galaxy catalog. Finally, the results as a whole are discussed and summarized in Sections 4 and 5 respectively. Throughout this study, we assume a $\Lambda \mathrm{CDM}$ cosmology with parameters $h=0.7 \mathrm{Mpc}^{-1}, \Omega_{\Lambda}=0.73$, and $\Omega_{M}=0.27$ (Planck Collaboration et al. 2014).

\section{ALMA Observations}

\subsection{Sample and Methods}

Our sample consists of deep $\sim 1.2 \mathrm{~mm}$ ALMA observations (Band 6) of five luminous objects at $z>6$. We use observations of two starbursts, CLM1 and WMH5 (Willott et al. 2015b), and two quasars, CFHQSJ 0210-0546 and J2329-0301 (Willott et al. 2013), as well as the data retrieved from the archive for an additional quasar, J054-0005 (Wang et al. 2013). There is archival data for other quasars observed in the Wang et al. (2013) study; however, the rms noise is larger $\left(\mathrm{rms}>0.5 \mathrm{mJy}\right.$ channel $^{-1}$ ), and there is a larger spectral resolution of $\sim 80 \mathrm{MHz}$; thus, it is not possible to detect companions within the luminosity range of interest to this study. For this reason, we have chosen not to include those fields in this study. For the first four data cubes (Willott et al. $2013,2015 b$ ), we analyze the full $\sim 8 \mathrm{GHz}$ from the four base bands, two centered on the extreme object and two spaced $\sim 15 \mathrm{GHz}$ away (in the upper sideband). In the archival data cubes from Wang et al. (2013), we were only able to retrieve the $2 \mathrm{GHz}$ baseband containing the quasar itself and thus have less continuum sensitivity and frequency bandwidth to search for companions. All of these data were obtained between 2012 and 2014, and we refer the reader to the papers cited for full information about observing strategies. The raw data from the archive was reimaged using the Common Astronomy Software Applications package (CASA v. 4.2.2; Mcmullin et al. 2007) task clean using the parameters suggested in the 
Table 1

Properties of Fields Searched for Companions

\begin{tabular}{|c|c|c|c|c|}
\hline Source Targeted & Frequency Coverage $(\mathrm{GHz})$ & rms Noise $^{\mathrm{a}}(\mathrm{mJy})$ & Beam Size & Reference \\
\hline$\overline{\text { CLM1 }}$ & $249.3-252.9,264.3-267.9$ & 0.18 & $0 . ! 5 \times 0.15$ & Willott et al. (2015b) \\
\hline WMH5 & $253.0-256.8,268.0-271.8$ & 0.22 & $0 " .5 \times 0 . " 5$ & Willott et al. (2015b) \\
\hline J0210-0456 & $254.7-256.2$ & 0.34 & $0 ! .79 \times 0 . .5$ & Willott et al. (2013) \\
\hline J2054-0005 & $269.2-270.9$ & 0.38 & $0 . " 57 \times 0 . " 51$ & Wang et al. (2013) \\
\hline
\end{tabular}

Note.

${ }^{a}$ Per $15 \mathrm{MHz}$ channel.

ScriptforImaging.py provided by the joint ALMA observatory along with the raw data. Once imaged, further analysis of the data cubes was performed with Python, relying on the spectralcube ${ }^{10}$ Python package.

The typical beam size of the observations is $\sim 0$ " 6 . Given that the sizes of known [C II] emitters at $z \sim 6$ are known to be $\lesssim 1^{\prime \prime}$ (Capak et al. 2015; Decarli et al. 2017), we do not expect any companions to be significantly spatially resolved. Our approach to selecting sources based on the peak flux recovers all [C II] emitters found in a survey of galaxies at $z=4.3$ (Miller et al. 2018). The sensitivities of these archival observations vary by a factor of 2 (listed in Table 1) but are on average deep enough to detect sources down to a $5 \sigma$ detection threshold of $L_{[\mathrm{C} \text { II] }} \approx 10^{8} L_{\odot}$ at $z=6$ for a Gaussian line profile with FHWM $=150 \mathrm{~km} \mathrm{~s}^{-1}$.

To search for line candidates in the ALMA data cubes, we developed a blind search algorithm. First, the entire cube was searched to find all points in the cube that exhibited a flux greater than $3 \times$ the rms noise in a single $15 \mathrm{MHz}$ channel (typically 0.75 mJy beam ${ }^{-1}$ ). With these positions recorded, the same positions in neighboring frequency slices were searched. If four surrounding channels (a minimal physical line width of $\sim 50 \mathrm{~km} \mathrm{~s}^{-1}$ ) had fluxes greater than $2 \times$ the rms noise (typically $0.5 \mathrm{mJy} \mathrm{beam}^{-1}$ ), the source was deemed a possible line candidate. The significance of these candidates was then investigated. The velocity FWHM of the candidate was measured by fitting a Gaussian, and a moment-zero map was constructed using the channels contained within the FWHM of the candidate. The signal-to-noise ratio $(\mathrm{S} / \mathrm{N})$ was calculated by dividing the integrated flux of the line by the average rms of the moment-zero map encompassing the FWHM. In this process, we make sure to mask the region of the map containing the primary target. Any object with an $\mathrm{S} / \mathrm{N}$ greater than 5 that lies within the FWHM of the primary beam is deemed a possible candidate. The possible candidates are inspected by hand to ensure that they show Gaussian-like line profiles. Four objects were identified by the algorithm with $\mathrm{S} / \mathrm{N}$ ratios greater than 5 , and all passed the inspection. These objects are presented as the companions discussed in the results section below. To further test the algorithm, we lowered the $\mathrm{S} / \mathrm{N}$ cutoff to 4 , identifying a further 16 candidates. These objects generally exhibited lower FHWM and peak fluxes than the $\mathrm{S} / \mathrm{N}>5$ sources. We ran additional tests on the full sample of $S / N>4$ lines to test their purity, as described in the subsection below.

\subsubsection{Purity of Sample}

Although our line candidates have an $\mathrm{S} / \mathrm{N}$ greater than 5 , it is still possible that they could be spurious detections due to the non-Gaussian phase noise of the interferometer or the large

\footnotetext{
${ }^{10} \mathrm{https}: / /$ spectral-cube.readthedocs.io/en/latest/
}

number of independent measurements made during our procedure (see Hayatsu et al. 2017, 2019). To estimate the rate at which false positives could occur, we apply our search algorithm to find negative peaks in the data. At an $\mathrm{S} / \mathrm{N}$ less than 5, we find that negative peaks at the same $S / N$ have similar distributions and properties as positive peaks but small FWHM values and are thus likely unphysical given their fluxes. However, there was only one negative peak with $\mathrm{S} / \mathrm{N}>$ 5 (in the CLM1 cube). This suggests that one of the four line candidates is a false positive. This false-positive rate, $25 \% \pm$ $25 \%$, is consistent with the statistical analysis performed by Aravena et al. (2016) on the ASPECS field; they predicted a false-positive rate of $\sim 35 \%$ for an $\mathrm{S} / \mathrm{N}$ cutoff of 5 . An additional possibility is that we are observing a peak in the dirty beam structure from the brighter target source. By analyzing the synthesized beam output of the clean function in CASA in each case, we find no strong side-lobe structures (dirty beam side-lobe peaks $\lesssim 8 \%$ of the central beam) at the positions of the candidates, suggesting that this is not a concern for the strong levels that we detect our candidate [C II] emitters. Further, the significant velocity offsets of our candidates from the central sources make it even more unlikely that they are related to the central source beam structure.

Even if the sources are real, we still must consider the possibility that these lines represent other transitions or species at different redshifts, the most likely being the mid- $J$ $\mathrm{CO}$ transitions. The $\mathrm{CO}(3-2), \mathrm{CO}(4-3)$, and $\mathrm{CO}(5-4)$ transitions are observable within the same frequency band at approximate redshifts of $0.3,0.8$, and 1.2 , respectively. Based on the Popping et al. (2016) models for the luminosity functions (LFs) of the $\mathrm{CO}$ rotational lines at various redshifts, we naively expect to see 0.25 sufficiently luminous lowredshift $\mathrm{CO}$ line-emitting galaxies in the volume spanned by the five cubes. This calculation takes into account the rms of each field and varying rms as a function of radius due to the ALMA primary beam. As the expected number of interlopers is $\ll 1$, we can safely neglect this as a possibility. Even though we are probing down to low flux values where the density of interlopers is higher, the volume spanned by our cubes is small enough that the number of interlopers expected is low. The predictions for the LFs from the Popping et al. model for the transitions and redshifts of interest agree well with current observational constraints (see the results of the 1 $\mathrm{mm}$ survey in Figure 4 of Decarli et al. 2016). However, these models underpredict the number of gas-rich galaxies at $z>1$ (Decarli et al. 2016). If future constraints show that the predicted LFs underestimate the number of bright sources for the transitions of interest, the number of expected interlopers will increase. 


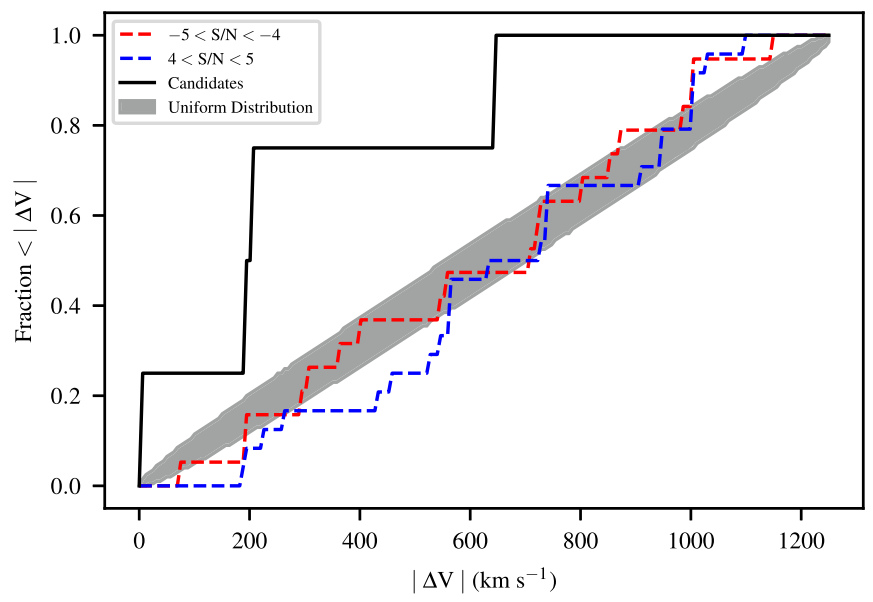

Figure 1. This figure displays the cumulative distribution of velocity offsets between the candidate line emitters and the primary targets, along with the expected distribution if the candidates were uniformly distributed in the data cubes. The gray band shows the 5\%-95\% confidence interval of the uniform distribution, calculated through bootstrapping. Through a Monte Carlo analysis, we find that the candidates are biased to a lower $\Delta V$ with respect to the uniform distribution at a $2 \sigma$ significance level. Also shown is the velocity offset distribution of positive and negative lines with $4<\mathrm{S} / \mathrm{N}<5$. They appear to have a more uniform distribution, unlike the $\mathrm{S} / \mathrm{N}>5$ candidates, which appear to be biased to lower velocities.

Figure 1 displays the cumulative distribution of the velocity offsets between the candidates and the primary ALMA targets, as well as the expected distribution if the candidates are uniformly distributed in the data cubes. The sidebands for the CLM1 and WMH5 cubes are not shown in Figure 1, as the velocity offset reaches $>10,000 \mathrm{~km} \mathrm{~s}^{-1}$, and it is difficult to show these alongside the smaller offsets. It is worth noting that there were no candidates found in these sidebands. It appears that the candidate distribution is inconsistent with a uniform distribution and the candidates are biased toward being closer to the central galaxies. This reinforces the idea that the candidates are real galaxies that are physically associated with the primary targets, as one would expect interlopers or spurious detections to be uniformly distributed in the cube. To test this, we perform a Monte Carlo analysis by repeatedly sampling four elements from the expected distribution to calculate the probability that all four randomly selected elements would have a lower $\Delta V$ than the maximum of the candidates $\left(642 \mathrm{~km} \mathrm{~s}^{-1}\right.$ for CLM1-A). This calculation includes the sidebands for the CLM1 and WMH5 cubes that are not shown in Figure 1. After 10,000 iterations, we find that $95 \%$ of the realizations contain at least one of the randomly selected velocity offsets, $\Delta V$, that is larger than the maximum of the candidates. Although this is only a marginally statistically significant detection $(\sim 2 \sigma)$, it is consistent with the candidates more likely appearing closer to the central galaxies with respect to a uniform distribution in the data cubes. As the velocity offset of CLM1-A $\left(642 \mathrm{~km} \mathrm{~s}^{-1}\right)$ is significantly larger than that of the next-highest candidate, J0210-0546-B at $205 \mathrm{~km} \mathrm{~s}^{-1}$, we also investigate the likelihood of finding three candidates within $205 \mathrm{~km} \mathrm{~s}^{-1}$. This is even less likely, with $99.6 \%$ of the realizations containing at least one of three candidates with $\Delta V>205 \mathrm{~km} \mathrm{~s}^{-1}$, a result that is statistically significant at the $\sim 3 \sigma$ level. This does not necessarily confirm the reality of our sources but simply indicates that their $\Delta V$ distribution is inconsistent with being uniformly distributed within the cubes.

\subsubsection{Voxel Flux Distribution}

In Figure 2, we further examine the noise properties of our ALMA data cubes. We plot the distribution of $60 \mathrm{MHz}$ voxel (data-cube pixel) flux values for each data cube, along with a Gaussian fit and the corresponding residuals. Since these are targeted observations, and we have not masked or removed any sources, we expect the positive side of the distribution to be skewed. Therefore, we focus on the distribution of voxels with negative fluxes. The distributions in each field are well represented by a Gaussian, showing residuals of less than one part in 50 for the $\pm 3 \sigma$ range. There appears to be an excess of voxels with negative flux in the $-4 \sigma$ to $-5 \sigma$ range compared to the overall Gaussian distribution. The excess of voxels with large negative fluxes is concerning, as they are likely caused by correlated, non-Gaussian noise. As these correlated noise spikes are equally likely to produce voxels with positive flux, it is possible that they could be mistaken for line emitters and produce false positives in our sample. We have verified that the non-Gaussianity is not caused by increased noise per channel near the edge of the sideband. By plotting the distribution of voxels excluding the upper and lower $25 \%$ of frequency slices in each cube, we observe a similar excess of voxels outside the $\pm 3 \sigma$ range.

\subsection{Results}

Upon inspection, we notice that one of the four candidates found by applying our search algorithm to the ALMA data sets is the source WMH5-B, previously discussed in Willott et al. (2015b). Willott et al. concluded that it is likely an ongoing merger with the more massive central galaxy WMH5-A. They concluded that it cannot be classified as two distinct sources; therefore, we do not consider WMH5-B for the following analysis.

Figure 3 displays three new line candidates used in the following analysis. The 1D spectrum, continuum, and line maps for each line candidate are shown. The channel map is extracted using the FWHM of the given line profile. The continuum map for each is constructed by using the frequency band containing the $[\mathrm{CII}]$ line, making sure not to include the frequency slices containing the line itself. These values are listed in Table 2. For the CLM1-A companion, we have additional data in the neighboring sidebands. We additionally investigate these sidebands for continuum emission from CLM1-A but again do not find a significant detection with $\mathrm{S} / \mathrm{N}<1$. A Gaussian function was fit to each line in order to extract a redshift, as well as the integrated flux and FWHM of the line. The observed properties of the three candidates are shown in Table 2.

The physical properties of the line candidates are listed in Table 2. The FWHM values of the four candidate lines range from 75 to $189 \mathrm{~km} \mathrm{~s}^{-1}$; the line luminosity, $L_{[\mathrm{C} \text { II] }}$, ranges from $7 \times 10^{7}$ to $2.5 \times 10^{8} L_{\odot}$ (corresponding to a range of integrated line fluxes of $0.07-0.25 \mathrm{Jy} \mathrm{km} \mathrm{s}^{-1}$ ); and only one of our line candidates is detected in the continuum at an $\mathrm{S} / \mathrm{N}>2$, with the remaining two showing $\mathrm{S} / \mathrm{N}<1$ continuum maps.

The observed properties of the candidate line emitters and primary galaxies are compared to previous detections of [C II] emission in high-redshift galaxies in Figure 4. The figure plots [C II] FWHM versus $L_{[\mathrm{C} \text { II] }}$ for the candidates and central sources in this study, detections of [C II] in $z \sim 5$ LBGs from Capak et al. (2015), and the companions and central QSOs 


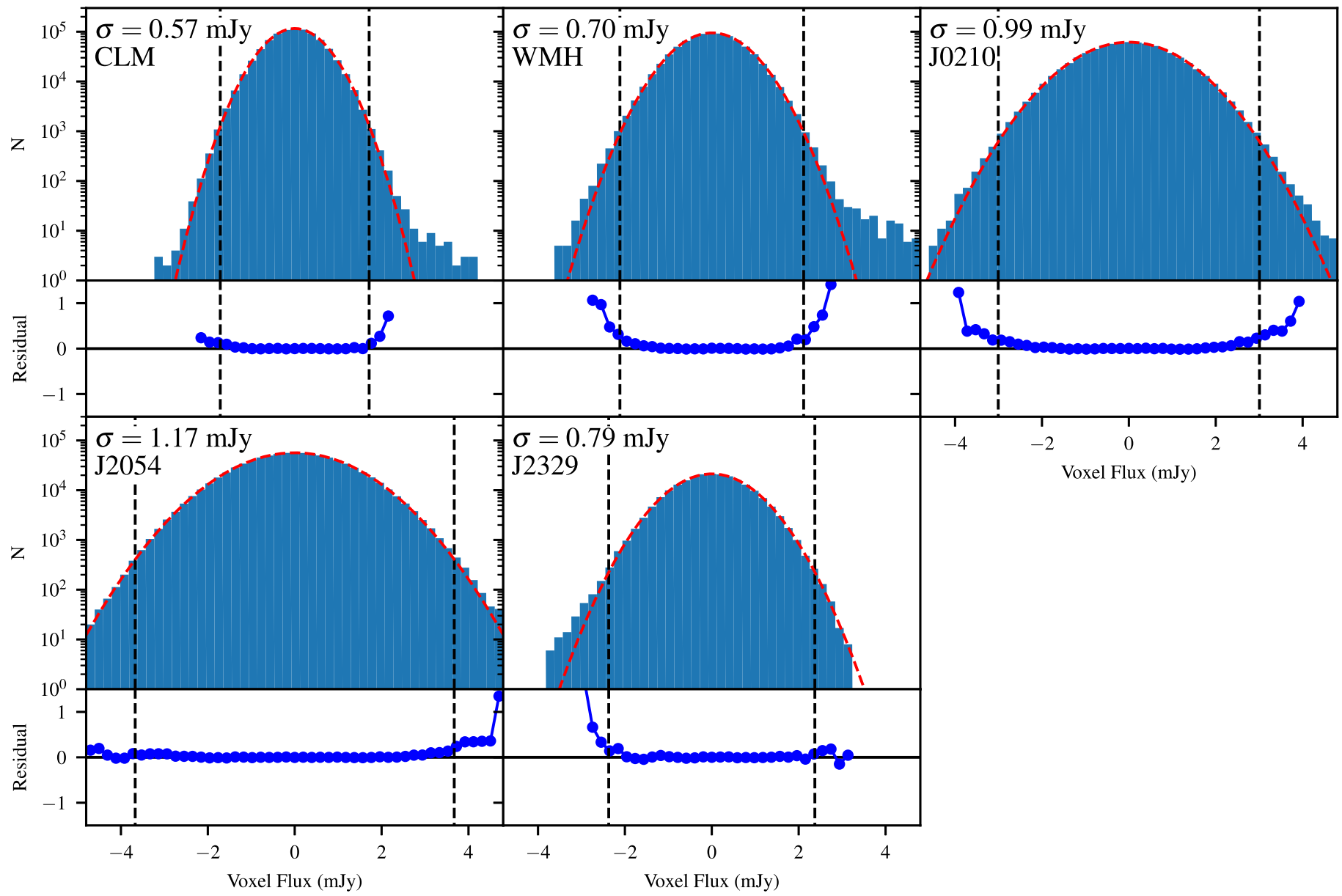

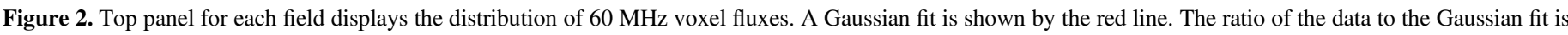

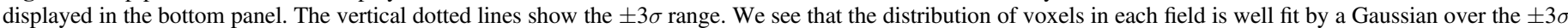

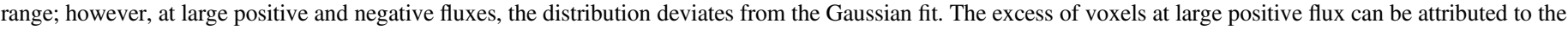
sources targeted in each field, but the voxels with large negative flux are likely caused by correlated noise spikes.

discussed in Decarli et al. (2017). The dotted line shows, based on our search algorithm, the minimum luminosity needed to reach an $\mathrm{S} / \mathrm{N}$ of 5 for a given FWHM value. This assumes a Gaussian line profile and the typical noise of our cubes $\left(\mathrm{rms} \sim 0.25 \mathrm{mJy}\right.$ channel $\left.^{-1}\right)$. Our candidates follow a similar distribution to the Capak et al. galaxies and Decarli et al. companions, extending the apparent relation to slightly lower values of $L_{[\mathrm{C} \text { II] }}$ and FWHM. None of our candidates appear as outliers in the distribution of known [C II] emitters. One might expect false-positive detections to have higher $L_{[\mathrm{C} \mathrm{II]}}$ at a given FWHM and thereby trace the detection threshold more closely. Moreover, the small volumes probed by our survey suggest that any companions we did find would be low-luminosity, relatively close to our detection threshold.

We can calculate the LFs of [C II] emitters in the observed fields used in this study. We integrate the volume possible to detect a line-emitting galaxy at a given luminosity by taking into account the differing noise properties of each field, as well as the effect of the ALMA primary beam (assumed to be a Gaussian with FWHM $=23^{\prime \prime}$ at this frequency). The redshift range covered is found through the spectral coverage of the ALMA data cubes, found in Table 1, and the known rest-frame emission of the [C II] line at $157.7 \mu \mathrm{m}$. The volume used to calculate the LF is thus different for each luminosity bin. Lower-luminosity galaxies cannot be detected to as large a radial distance as higher-luminosity galaxies due to the effect of the primary beam on the noise amplitude in the data cube; thus, the volume probed is larger for highluminosity galaxies.

The LFs of [C II] emitters at $z \sim 6$ from our study, as well as other recent measurements and predictions, are displayed in Figure 5. Our data are shown both including and neglecting the primary targets of the ALMA observations. Where we only find one companion, we treat our data as an upper limit. Also shown are various measurements of the field [C II] LF at $z \sim 6$ (Swinbank et al. 2012; Aravena et al. 2016; Yamaguchi et al. 2017). The most constraining field measurement comes from the ASPECS survey (Aravena et al. 2016), which was calculated through a blind search for [C II] lines along with searching at the positions of known optical dropouts. It is worth noting that this measurement, along with others, is formally an upper limit, as the reality of all of their candidates still needs to be confirmed. With future work, these constraints could change significantly. Also shown are theoretical predictions of the [C II] LF from previous studies (Popping et al. 2016; Lagache et al. 2018) along with a prediction from this study based on the Hayward et al. (2013a) semianalytic model (SAM; see Section 3). While it appears that the theoretical predictions underestimate the observations, especially the Popping et al. (2016) prediction, which drops precipitously at $L_{[\mathrm{C} \mathrm{II}]}>10^{8} L_{\odot}$, most of the 


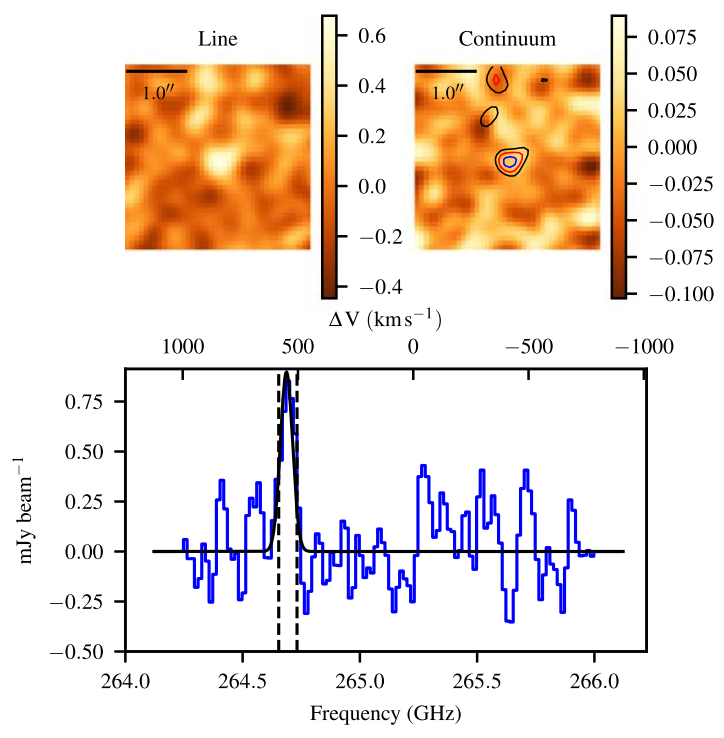

(a) CLM1-A

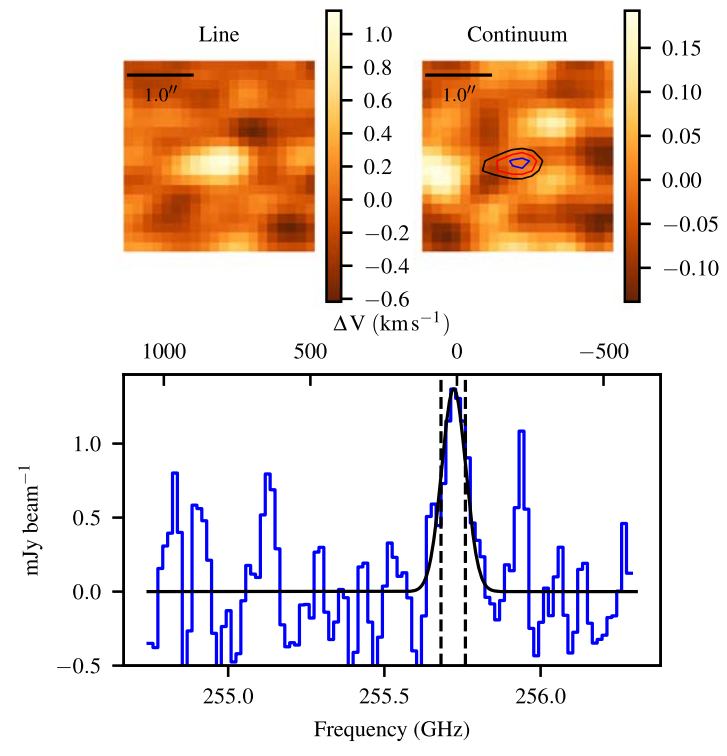

(b) CFHQS J0210-A

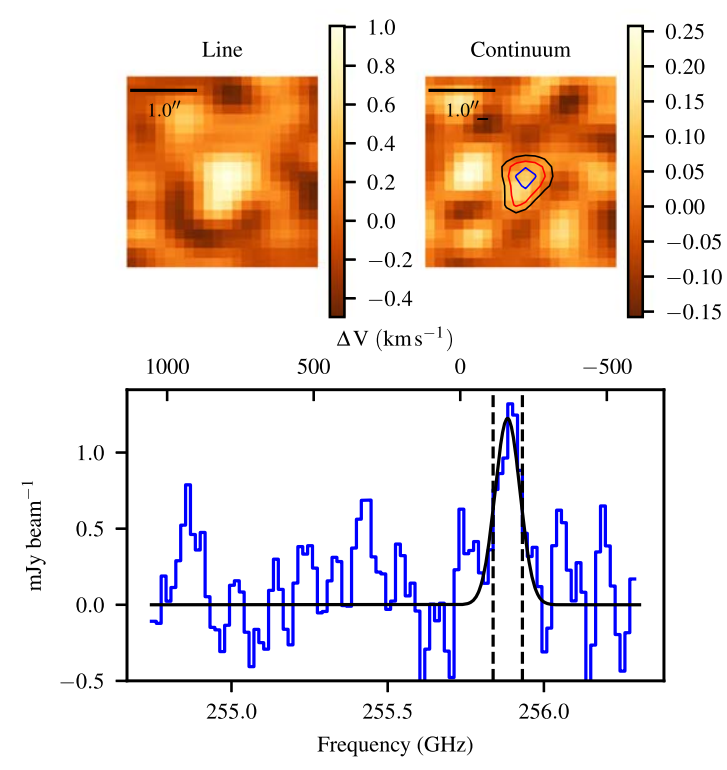

(c) CFHQS J0210-B

Figure 3. Four line candidates found by the blind search of the deep ALMA cubes are shown here. A Gaussian fit to the 1D spectra is shown in black, with the vertical dotted lines denoting the FWHM of the line. The bottom axis shows the frequency of observations, while the top displays the velocity offset from the primary ALMA target. We show the line flux, calculated using only the frequency slices within the FWHM of the line, as well as the continuum flux. Contours on the continuum images represent 0.9 (blue), 0.7 (red), and 0.5 (black) times the peak flux in the corresponding line channel. (a) Candidate found near the UV-luminous LBG CLM1. ALMA data were originally taken by and analyzed in Willott et al. (2015b). (b) Candidate found near the quasar J0210-0456, originally analyzed by Willott et al. (2013). (c) Second candidate found near the quasar J20210-0456.

observational constraints formally represent upper limits. If, indeed, only one of the sources from the Aravena et al. (2016) survey is real, then the measured density of sources at $L_{\text {[C II] }}>3 \times 10^{8} L_{\odot}$ is only a factor of $\sim 3$ discrepant with the Lagache et al. prediction.

At $L_{[\mathrm{C} \text { II] }} \sim 10^{8} L_{\odot}$, we find our measurement of the LF to be larger than any other measurement or theoretical prediction. Specifically, when comparing the density of all sources (candidates presented in this paper and primary targets) at $L_{\text {[C II] }}>10^{8} L_{\odot}$, we find a relative overdensity of $12_{-6}^{+7.5}$ when comparing to the Aravena et al. (2016) measurement extrapolated to lower luminosity. However, this is likely a lower limit on the overdensity, and comparing to the Lagache et al. (2018) prediction, we find a larger relative overdensity of $158_{-79}^{+104}$. Given that these are targeted observations, the interpretation of this relative overdensity is difficult. In an attempt to correct for this, we also consider the LF of just the candidate companions presented in this paper. For only the companions, we calculate relative overdensities of $6_{-3}^{+4}$ and $86_{-47}^{+60}$ at $L_{[\mathrm{C} \mathrm{II}]}>10^{8} L_{\odot}$ when comparing to the Aravena et al. (2016) observation and Lagache et al. (2018) prediction, respectively. 


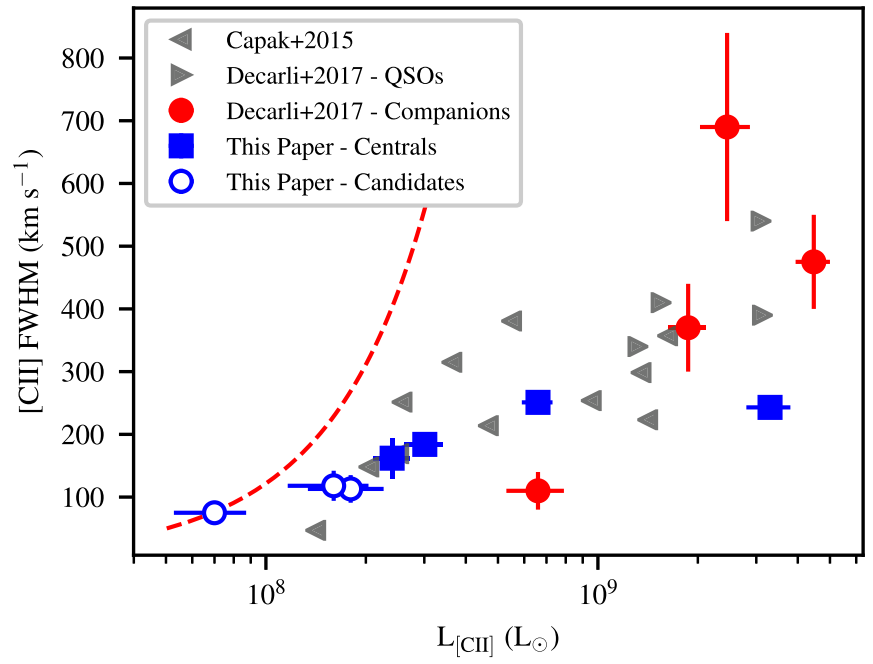

Figure 4. This figure displays our line candidates compared to the Capak et al (2015) sample of $z \sim 5$ LBGs and Decarli et al. (2017) sample of quasars and associated companions in the $[\mathrm{CII}]$ FWHM vs. $L_{[\mathrm{CII}}$ plane. The red line shows, for a given FWHM value, the minimum luminosity needed to achieve an $\mathrm{S} / \mathrm{N}$ of 5. This is based on a Gaussian line profile and typical noise in our cubes $(\mathrm{rms} \sim 0.25 \mathrm{mJy})$. Our line candidates and primary galaxies follow a similar distribution to the previously observed galaxies, with our candidates extending an apparent relation to slightly lower $L_{[\mathrm{C} \mathrm{II]}}$ and FWHM but lying significantly above our minimum detection threshold line.

\section{Simulations of [C II] Emitters around the Most Luminous Galaxies in the EoR}

\subsection{Mock Galaxy Catalogs}

To help interpret the results shown in Section 2 we employ mock galaxy catalogs described in Hayward et al. (2013a), where we parameterize the galaxies primarily by their observed $L_{\text {[C II] }}$ and $850 \mu \mathrm{m}$ continuum fluxes. We provide a brief description of the methodology here but refer the reader to the original paper for full details. Using a halo catalog from the Bolshoi simulation, eight mock light cones from $0.5<z<$ 8 are constructed by starting at random locations and choosing a random sight line (Klypin et al. 2011; Behroozi et al. $2013 b, 2013 c)$. The eight mock galaxy catalogs cover a total area of $15.7 \mathrm{deg}^{2}$ extending out to $z=8\left(1^{\circ} .4\right.$ by 1.4 for each field). Stellar masses and SFRs are assigned to halos based on their mass and redshift using the functions derived in Behroozi et al. (2013a) from subhalo abundance matching. ${ }^{11} \mathrm{We}$ then assign [C II] luminosities to galaxies in the catalog based on the power-law scaling between SFR and $L_{\text {[C II] }}$ empirically found in De Looze et al. (2014) applied with 0.42 dex of scatter, as quoted in their study. There is clearly uncertainty in the relation between [C II] luminosity and SFR during the EoR, yet this simple power-law scaling, empirically derived in the local universe, has been shown to match observations fairly well (Capak et al. 2015; Vallini et al. 2015). The $850 \mu \mathrm{m}$ flux densities $\left(S_{850}\right)$ are assigned following Hayward et al. $(2013 \mathrm{~b})$ : dust masses are computed using empirical scaling relations between gas fraction, metallicity, and stellar mass. Then, $S_{850}$ is assigned based on a fitting function using SFR and dust mass, which was derived by performing dust radiative transfer calculations on hydrodynamical simulations of galaxy mergers and isolated disk galaxies.

\footnotetext{
${ }^{11}$ We note that these prescriptions have recently been updated in Behroozi et al. (2019).
}

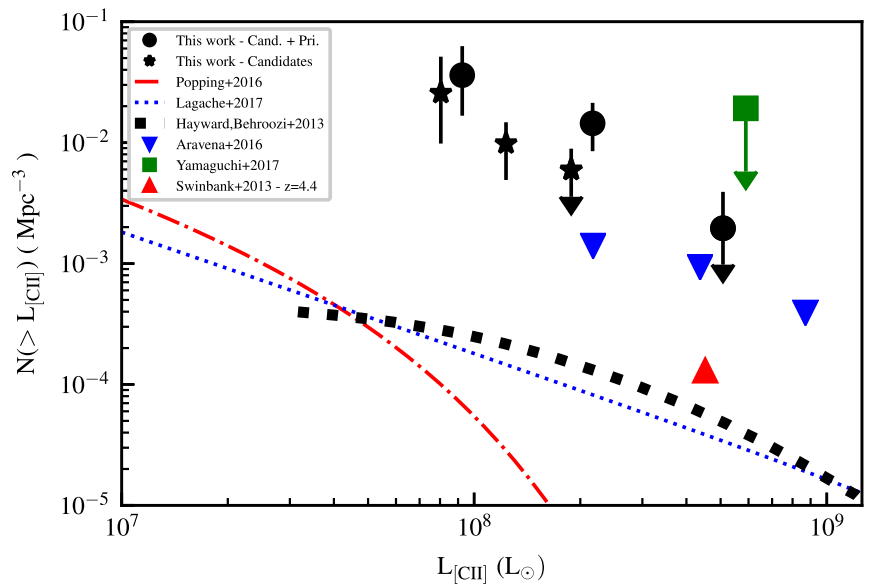

Figure 5. Plot displaying various measurements and predictions for the LFs of $[\mathrm{C}$ II] emitters at $z=6$. The black squares display the density of all sources the fields used in this study, while the black stars show the LF only considering the three new line candidates discussed in this study. The blue triangles show recent observational constraints made by Aravena et al. (2016) at $6<z<8$, and the red triangle shows a measurement by Swinbank et al. (2012) at $z=4.4$. Observational constraints from Yamaguchi et al. (2017) are also shown by a green square. Predictions for the $z=6$ [C II] LF based on semianalytic models discussed in Popping et al. (2016) and Lagache et al. (2018) are shown by the dotted and dotted-dashed lines, respectively. Hayward et al. (2013a) displayed a prediction from an abundance-matching model combined with the empirical SFR $-L_{[\mathrm{C} \text { II] }}$ relation from De Looze et al. (2014). Section 3 contains a full description of the Hayward et al. model. While the [C II] LF is not well constrained at $L_{[\mathrm{C} \mathrm{II}]} \sim 10^{8} L_{\odot}$, the LFs of candidates in the fields used lie at least an order of magnitude above any measurement or prediction. This suggests that luminous galaxies $z>6$ represent biased regions and therefore signpost overdensities in the early universe.

Table 2

Displaying the Properties of the Three New Line Candidates Found by Using the Blind Search Algorithm Described in Section 2.1

\begin{tabular}{lccc}
\hline \hline Source Name & CLM1-A & $\begin{array}{c}\text { CFHQSJ } \\
\text { 0210-0546-A }\end{array}$ & $\begin{array}{c}\text { CFHQSJ } \\
0210-0546-B\end{array}$ \\
\hline R.A. (J2000) & $2: 28: 02.970$ & $2: 10: 13.883$ & $2: 10: 13.501$ \\
Decl. (J2000) & $-4: 16: 11.74$ & $-4: 56: 22.86$ & $-4: 56: 19.26$ \\
$z_{\text {[C II] }}$ & 6.180 & 6.432 & 6.427 \\
$\Delta v\left(\mathrm{~km} \mathrm{~s}^{-1}\right)$ & 642 & -2 & -191 \\
Proj. sep. $(\mathrm{kpc})$ & 37 & 58 & 27 \\
Peak flux (mJy) & 0.861 & 1.19 & 1.320 \\
Integrated line flux & $0.07 \pm 0.01$ & $0.16 \pm 0.03$ & $0.15 \pm 0.03$ \\
$\quad\left(\mathrm{Jy} \mathrm{km} \mathrm{s}{ }^{-1}\right)$ & $75 \pm 14$ & $113 \pm 22$ & $118 \pm 24$ \\
FWHM $\left(\mathrm{km} \mathrm{s}^{-1}\right)$ & $0.7 \pm 0.2$ & $1.8 \pm 0.5$ & $1.6 \pm 0.4$ \\
$L_{[\mathrm{C} \text { II] }}\left(10^{8} L_{\odot}\right)$ & 5.01 & 5.12 & 5.04 \\
Line S/N & 0.77 & 0.42 & 2.27 \\
Continuum S/N & & & \\
\hline
\end{tabular}

\subsection{Simulation Results}

To directly compare the simulations with the observed counts from the ALMA data, we take the fields surrounding the simulated galaxies with $L_{[\mathrm{C} \text { II] }}$ matched to that of the primary ALMA targets $\left(L_{[\mathrm{C} \mathrm{II}]}=5 \times 10^{8}-10^{9} L_{\odot}\right)$ at $6<z<6.5$. We search the simulation around these galaxies for companions within a $15^{\prime \prime}$ radius and $d z=0.05$, comparable to the search volume of the ALMA observations. Figure 6 shows the number of companions above a given [C II] luminosity for the simulated fields along with the observed ALMA fields from Section 2. The simulated field counts derived from the total $15.7 \mathrm{deg}^{2}$ are also displayed. 


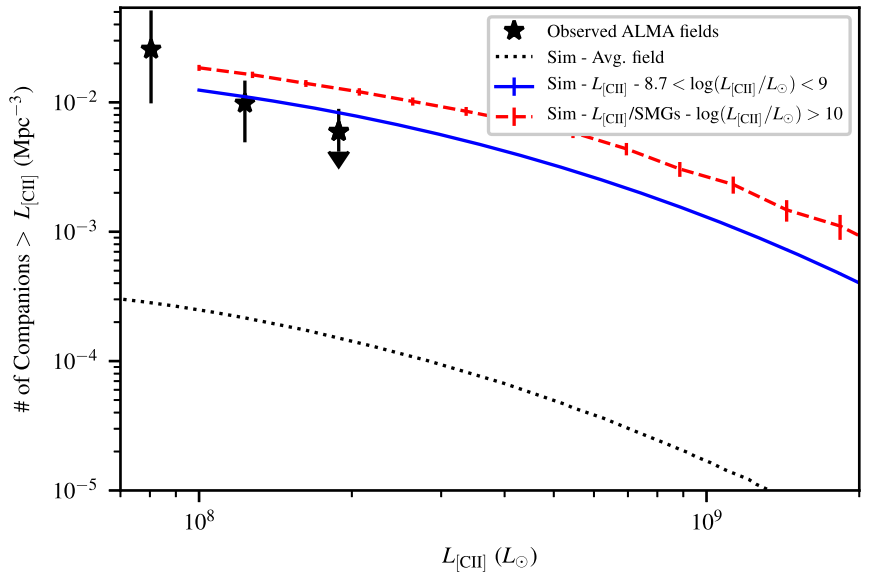

Figure 6. We show the $[\mathrm{C} \mathrm{II}]$ LF measured from our simulation and the ALMA fields from Section 2. The blue lines shows the LFs of companions surrounding simulated galaxies with $L_{[\mathrm{C} \text { II] }}$ matched to the central galaxies of the ALMA observations $\left(L_{[\mathrm{C} \mathrm{II}]}=0.5-1 \times 10^{9} L_{\odot}\right)$. Our observational constraints, described in Section 2 are shown as black stars. The red line shows the density of companions around the most luminous [C II]-emitting galaxies in the simulation, most of which would be observable as SMGs. The black line shows the field measurement of the simulations, calculated using the entire simulation volume available. We find that the simulation and observations show good agreement, reinforcing the idea that luminous galaxies at $z>6$ represent biased regions.

We find a relative overdensity of [C II] companions around the matched $L_{[\mathrm{C} \text { II] }}$ sample of simulated galaxies of $50 \pm 1$, consistent with the observational result. There is good agreement between the simulations and observations of the number of companions in fields surrounding galaxies of the same [C II] luminosity as the observed primary ALMA targets. The density of companions, although enhanced compared to the field measurement, follows a similar shape as the field LF. It is worth noting that the simulation is incomplete at $L_{\text {[C II] }} \lesssim 10^{8} L_{\odot}$ due to the minimum halo mass in the catalog.

We also show the counts surrounding the galaxies with the highest [C II] luminosities in the simulation: $L_{[\mathrm{C} \mathrm{II}]}>10^{10} L_{\odot}$. These represent the most luminous simulated galaxies at this epoch and could represent the highest overdensities that are forming stars rapidly, with SFRs comparable to SMGs. This high-luminosity sample of simulated galaxies consists of two populations: those with high intrinsic SFR $\left(>100 M_{\odot} \mathrm{yr}^{-1}\right)$ and those with lower intrinsic SFR that have elevated $L_{\text {[C II] }}$ due to the scatter in the $L_{[\mathrm{C} \mathrm{III}]}-\mathrm{SFR}$ relation. The former group generally has $S_{850}>1.5 \mathrm{mJy}$ and would be detected as SMGs by current and upcoming facilities. These extremely luminous simulated galaxies contain 1.5 times the number of companions as the matched $L_{[\mathrm{C} \mathrm{II}]}$ sample of simulated galaxies. Therefore, by investigating the most luminous [C II] emitters in the simulation, we find even more biased regions.

Given that we have full information available in the mock catalog, we are able to investigate whether the regions around luminous [C II] emitters during the EoR signpost peak in the large-scale matter distribution. Figure 7 shows the total dark matter mass in a volume surrounding the matched and highest $L_{[\mathrm{C} \text { II] }}$ samples along with random locations over a redshift range of $5.5<z<7$. The volume used to calculate the total dark matter mass is defined by $2^{\prime} \times 2^{\prime}$, with $d z=0.2$ corresponding to a volume of roughly $2000 \mathrm{cMpc}^{3}$. While this is larger than the volume probed by typical ALMA observations, our goal is to test the connection of [C II]-emitting galaxies at $z \sim 6$ to the large-scale distribution of matter at this

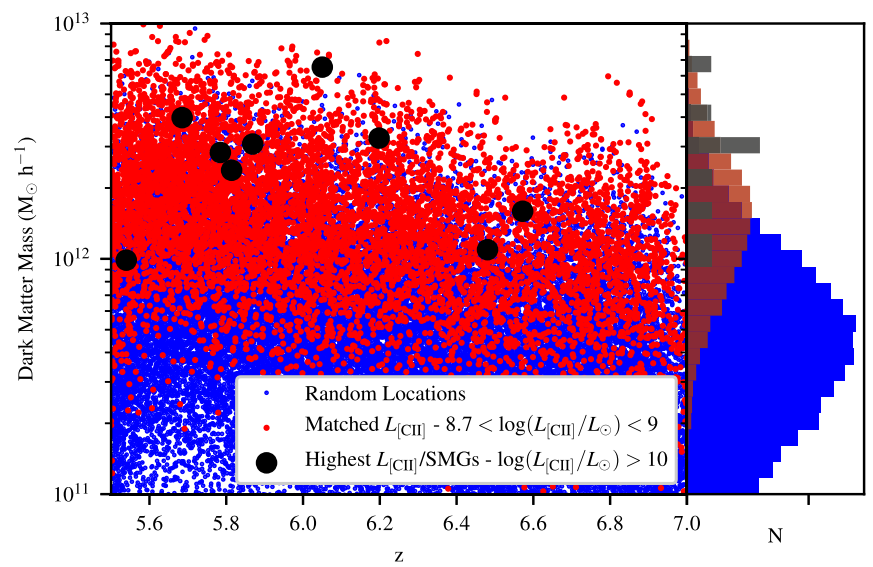

Figure 7. This figure displays dark matter mass in an $\sim 200 \mathrm{cMpc}^{3}$ volume centered on a given galaxy vs. redshift. Dark matter mass is calculated by summing the mass of all of the halos in a given region. We show the matched [C II] luminosity sample along with the most luminous [C II] emitters or SMGs. The histogram shows the total distribution of dark matter masses for each sample in the range $5.5<z<7$ along with the distribution of randomly located regions. The matched $L_{[\mathrm{C} \text { II] }}$ samples often reside in overdensities, but there is a large scatter in the mass, while the highest $L_{[\mathrm{C} \mathrm{II}]} / \mathrm{SMG}$ samples consistently reside in the most massive regions during the EoR.

epoch. This approach was used by Miller et al. (2015) to investigate the bias and clustering of SMGs at $z \sim 2$.

The regions surrounding the matched $L_{[\mathrm{C} \text { II] }}$ sample of galaxies tend to have larger dark matter masses with a mean mass of $10^{12.1 \pm 0.3} M_{\odot}$ compared to $10^{11.5} M_{\odot}$ for the random sample. However, due to the scatter, some simulated galaxies in the matched luminosity range lie in relatively underdense regions of space, while some live in the most massive regions $\left(M>5 \times 10^{12} M_{\odot}\right)$. By contrast, the most extreme $L_{[\mathrm{C} \mathrm{II}]}$ emitters consistently lie in massive overdensities with a larger mean mass of $10^{12.4 \pm 0.2} M_{\odot}$ with a smaller scatter, and no regions have a total mass lower than $10^{12} M_{\odot}$.

\section{Discussion}

We have searched for companions in archival ALMA data targeting [C II] from known $z>6$ quasars and ULIRGs. Our analysis has revealed three new and one previously known companion galaxies in the five fields searched. Previously, WMH5-B was discovered and discussed in Willott et al. (2015b), providing validation of our method. The physical properties of our line candidates are comparable to those found by other ALMA studies targeting the [C II] line from known high-redshift galaxies (Capak et al. 2015); however, the statistical analysis of the purity of our sample suggests that at least one of our candidates may be a false positive. As the faintest galaxy in our sample (CLM1-A) lies near our selection limit (Figure 4) with a somewhat low luminosity for its FWHM, it may not be a real galaxy. It is also possible that this offset is simply due to scatter in the $L_{[\mathrm{C} \mathrm{II}}-\mathrm{FWHM}$ relation.

We are able to robustly detect lower-luminosity galaxies than other studies because the noise in these pointed deep fields is much lower than in larger-area surveys. Aravena et al. (2016) performed a blind survey for $z>6[\mathrm{C} \mathrm{II}]$ emitters in a blank field over a similar area (seven pointings covering $\sim 1 \operatorname{arcmin}^{2}$ ) but a much larger volume due to their seven frequency tunings over the ALMA Band 6. However, their average rms of $0.56 \mathrm{mJy}$ per $31.25 \mathrm{MHz}$ channel is about twice that of the data we employ. They found only $\sim$ one to two line candidates that 
lie within the physical region of FWHM- $L_{[\mathrm{C} \text { II] }}$ occupied by the galaxies in this study and that of Capak et al. (2015). They noted specifically that for this reason, in addition to their purity analysis, most of their candidates are probably not real. Despite the small angular size of our deep ALMA pointings, the biased regions have allowed us to uncover lower-luminosity galaxies than previously found due to their increased numbers in these overdense fields.

The lack of significant IR continuum detections for three of our candidates is not unexpected. Known galaxies at this epoch with $L_{[\mathrm{C} \text { II }]} \sim 10^{8} L_{\odot}$ have total IR luminosities of roughly $5 \times 10^{10} L_{\odot} \quad($ Capak et al. 2015). This corresponds to an observed $1.1 \mathrm{~mm}$ continuum flux of roughly $30 \mu \mathrm{Jy}$ (Casey et al. 2014), while the data cubes used have a typical $3 \sigma$ detection limit of $65 \mu \mathrm{Jy}$. Thus, our candidates would be undetected in the IR continuum if they followed these known relations. An IR luminosity exceeding $10^{11} L_{\odot}$ is needed for a galaxy to be detected in the continuum with $>3 \sigma$ confidence in the cubes used in this study. Similarly, only two of the four companions found in Decarli et al. (2017) have FIR continuum detections, yet they possess [C II] luminosities over an order of magnitude larger than the companions in this study. The lack of FIR continuum detections of the companions is therefore consistent with the known relation between [C II] and FIR luminosity at $z>6$.

We may also be able to use the lack of continuum detections to rule out the possibility that our candidates are interlopers at lower redshift. To assess this possibility, we consider three separate cases of $\mathrm{CO}$ transitions at lower redshift: $\mathrm{CO}(5-4)$ at $z=1.2, \mathrm{CO}(4-3)$ at $z=0.8$, and $\mathrm{CO}(3-2)$ at $z=0.3$. For each case, we take the average line flux of our candidates $\left(\sim 0.1 \mathrm{Jy} \mathrm{km} \mathrm{s}^{-1}\right)$ and calculate the inferred gas mass from each particular line assuming a Milky Way-like CO spectral line energy distribution and a conversion factor $\alpha_{\mathrm{CO}}=4$. Next, we infer an SFR from the gas mass using the disk model derived in Hayward et al. (2013b), assuming a size of $1 \mathrm{kpc}$. Finally, we calculate the inferred $1.1 \mathrm{~mm}$ flux density, $S_{1.1 \mathrm{~mm}}$, by assuming a conversion factor of $300 \frac{M_{\odot} \mathrm{yr}^{-1}}{\mathrm{mJy}}$ (Hayward et al. 2013b; Barger et al. 2014). We find the inferred $S_{1.1 \mathrm{~mm}}$ to be equal to $\sim 20$, $\sim 10$, and $\sim 5 \mu \mathrm{Jy}$ for each case, respectively. These are all under the detection threshold of the continuum data (roughly $65 \mu \mathrm{Jy}$ ); thus, the lack of detection cannot rule out the possibility of interlopers.

To further test the possibility of interlopers, we perform a search of publicly available near-IR (NIR) and optical data. We find no sources in Spitzer IRAC (CH1 and $\mathrm{CH} 2)$ images at the locations of any of the three companions; however, the integration times were quite short, only roughly $100 \mathrm{~s}$. We also do not find optical counterparts in either the $g, r$, or $z$ bands of the DECaLS DR8 images (Dey et al. 2019). Using the gas masses for the three cases above and the relationship between gas mass, stellar mass, and redshift shown in Hopkins et al. (2010), we estimate a stellar mass for the three cases of $\sim 8 \times 10^{8}, \sim 2 \times 10^{8}$, and $\sim 4 \times 10^{7} M_{\odot}$, respectively. These are very likely to be below the detection limit and therefore would go undetected in the available NIR and optical data. This is also the case if the candidates were at $z=6$. Again, the lack of NIR and optical counterparts does not constrain the possibility of our candidates being interlopers.

Since our ALMA sample is biased to fields around extreme objects at $z>6$, we are not able to directly constrain the field LF; however, we can make predictions about the clustering and bias of galaxies at this epoch (Figure 5). Even if the existing blank field surveys were extended to deeper flux limits comparable to our fields, we predict based on our counts/ overdensity analysis that these blank field surveys would not be large enough to uncover significant numbers of fainter sources. Based on the field LF function derived in Section 3, a survey the size of the ASPECS survey (see Aravena et al. 2016; $\sim 1 \operatorname{arcmin}^{2}$ ) would likely only detect one source at $L=10^{8}$ $L_{\odot}$. By comparison to our simulations, we find that the number counts in regions surrounding simulated galaxies with similar $L_{[\mathrm{C} \text { II] }}$ to the primary targets from the ALMA analysis show good agreement with the observed data. The factor of $86_{-47}^{+60}$ overdensity we measure from the ALMA data set is similar to what we find in the fields of extreme galaxies in the simulation and appears to reflect the underlying matter overdensity. We also note that the overall LF from the simulations, shown in Figure 5, shows fairly good agreement with another recent prediction of the [C II] field $\mathrm{LF}$ at $z=6$ by Lagache et al. (2018).

These results agree with a recent study by Decarli et al. (2017). They found that roughly $16 \% \pm 8 \%$ (four companions for 25 targets, assuming Poisson statistics) of quasars at $z>6$ host a nearby companion [C II] emitter. We find companions at a rate of $60 \% \pm 34 \%$ (three companions for five targets). Our companion rate appears slightly larger; however, we have adopted a lower significance and luminosity threshold (S/N $=5$ and $\sim 10^{8} L_{\odot}$, respectively). Decarli et al. adopted a stringent $7 \sigma$ cut and therefore only found companions with $L_{\text {[C II }]}>10^{9} L_{\odot}$. Given that we find no sources in this luminosity range, the $1 \sigma$ upper limit on our companion rate is $36 \%$ (or 1.8 out of five fields) at $L_{\text {[C II] }}>10^{9} L_{\odot}$, consistent with the findings of Decarli et al.

Additionally, we have shown through the use of the simulations that luminous [C II] emitters in the EoR not only possess an excess of companions compared to random fields but also represent overdensities in the large-scale matter distribution. The simulations inevitably have some limitations, and the apparent agreement with our ALMA observations should be measured with these caveats. In the mock galaxy catalogs, only star-forming galaxies parameterized by their FIR/submillimeter emission are adopted in this realization, and [C II] luminosity is assigned solely based on SFR. The quasar phase of galaxies and the growth of supermassive black holes are not specifically treated in this implementation. Thus, the connection to our three quasar fields is not entirely well motivated, although the starforming and quasar phases have often been shown to be tightly linked (e.g., Harrison et al. 2012a, 2012b).

\section{Conclusion}

We present a search for companion [C II] emitters around known luminous sources during the EoR. Using ALMA to observe [C II] emission allows us to overcome the shortcomings of other similar studies trying to observe overdensities at $z>6$ around rare and extreme sources. We develop an algorithm to search for companion [C II] line emitters in deep Band 6 ALMA data of previously observed luminous galaxies and quasars. A similar analysis is then performed on a mock galaxy catalog to put the ALMA results in context. The major results are as follows.

1. We find three new candidate companions from our blind search of deep ALMA data of known luminous galaxies and quasars. All candidates display a [C II] line $\mathrm{S} / \mathrm{N}$ 
greater than 5 and lie within a projected radius of $60 \mathrm{kpc}$ and $650 \mathrm{~km} \mathrm{~s}^{-1}$, supporting the idea that they are physically associated with the central galaxies.

2. The three candidates display similar physical properties to previously studied galaxies during the EoR. We find the same $L_{[\mathrm{C} \mathrm{III}}$ versus [C II] FWHM relation observed in Capak et al. (2015) and Decarli et al. (2017) extended to lower-luminosity values.

3. By calculating the LF of the central galaxies and the candidates, we quantify the overdensity. These luminous galaxies represent highly biased regions during the EoR. Even though there are few constraints on the LFs of [C II] emitters at $z>6$, our fields show a relative overdensity of companions of at least $6_{-3}^{+4}$ when comparing to observational constraints from Aravena et al. (2016) and $86_{-47}^{+60}$ when comparing to the prediction from Lagache et al. (2018).

4. By performing a similar analysis on a mock galaxy catalog, we find comparable results to the analysis of the ALMA fields. Matching the $L_{[\mathrm{C} \text { II] }}$ of the extreme sources in the simulation to the primary targets of the ALMA observations, we find a similar overdensity to the field population in the regions surrounding the simulated luminous [C II] emitters. Furthermore, the most luminous simulated [C II]-emitting galaxies $\left(L_{[\mathrm{C} \mathrm{II}]}>10^{10} L_{\odot}\right)$ host even more companions, by a factor of $\sim 1.5$.

5. By investigating the matter distribution around these sources in the simulation, we find that the luminous [C II] emitters during the EoR reside in overdense regions of space. This confirms that these simulated galaxies not only possess an excess of [C III]-emitting companions in their vicinity but also signpost peaks in the large-scale matter distribution.

We thank the anonymous referees, whose suggestions greatly improved the manuscript. This paper makes use of the following ALMA data: ADS/JAO.ALMA\#2013.1.00815.S., ADS/JAO. ALMA\#2011.0.00243.S, and ADS/JAO.ALMA\#2011.0.00206. S. ALMA is a partnership of the ESO (representing its member states), NSF (USA), and NINS (Japan), together with the NRC (Canada) and NSC and ASIAA (Taiwan), in cooperation with the Republic of Chile. The Joint ALMA Observatory is operated by the ESO, AUI/NRAO, and NAOJ. The National Radio Astronomy Observatory is a facility of the National Science Foundation operated under cooperative agreement by Associated Universities, Inc. T.B.M. would like to thank the Killam Trust and the Gruber Foundation for support. S.C.C. acknowledges the Killam Trust, NSERC, and CFI for support. Support for P.B. through program No. HST-HF2-51353.001-A was provided by NASA through a Hubble Fellowship grant from the Space Telescope Science Institute, which is operated by the Association of Universities for Research in Astronomy, Inc., under NASA contract NAS5-26555. The Flatiron Institute is supported by the Simons Foundation.

\section{ORCID iDs}

Christopher C. Hayward (1) https://orcid.org/0000-00034073-3236
Chris J. Willott (1) https://orcid.org/0000-0002-4201-7367

\section{References}

Aravena, M., Decarli, R., Walter, F., et al. 2016, ApJ, 833, 71 Bañados, E., Venemans, B. P., Walter, F., et al. 2013, ApJ, 773, 178 Barger, A. J., Cowie, L. L., Chen, C. C., et al. 2014, ApJ, 784, 9 Behroozi, P., Wechsler, R. H., Hearin, A. P., \& Conroy, C. 2019, MNRAS, 488, 3143

Behroozi, P. S., Wechsler, R. H., \& Conroy, C. 2013a, ApJ, 770, 57 Behroozi, P. S., Wechsler, R. H., \& Wu, H.-Y. 2013b, ApJ, 762, 109 Behroozi, P. S., Wechsler, R. H., Wu, H.-Y., et al. 2013c, ApJ, 763, 18 Bouwens, R. J., Aravena, M., Decarli, R., et al. 2016, ApJ, 833, 72 Capak, P. L., Carilli, C., Jones, G., et al. 2015, Natur, 522, 455 Casey, C. M., Narayanan, D., \& Cooray, A. 2014, PhR, 541, 45 Champagne, J. B., Decarli, R., Casey, C. M., et al. 2018, ApJ, 867, 153 Chiang, Y.-K., Overzier, R., \& Gebhardt, K. 2013, ApJ, 779, 127 De Looze, I., Cormier, D., Lebouteiller, V., et al. 2014, A\&A, 568, A62 Decarli, R., Walter, F., Aravena, M., et al. 2016, ApJ, 833, 12 Decarli, R., Walter, F., Venemans, B. P., et al. 2017, Natur, 545, 457 Decarli, R., Walter, F., Venemans, B. P., et al. 2018, ApJ, 854, 97 Dey, A., Schlegel, D. J., Lang, D., et al. 2019, AJ, 157, 168 Farina, E. P., Venemans, B. P., Decarli, R., et al. 2017, ApJ, 848, 78 Goto, T., Utsumi, Y., Kikuta, S., et al. 2017, MNRAS: Letters, 470, L117 Harrison, C. M., Alexander, D. M., Mullaney, J. R., et al. 2012a, ApJL, 760, L15

Harrison, C. M., Alexander, D. M., Swinbank, A. M., et al. 2012b, MNRAS, 426, 1073

Hayatsu, N. H., Ivison, R. J., Andreani, P., et al. 2019, RNAAS, 3, 97

Hayatsu, N. H., Matsuda, Y., Umehata, H., et al. 2017, PASJ, 69, 45

Hayward, C. C., Behroozi, P. S., Somerville, R. S., et al. 2013a, MNRAS, 434,2572

Hayward, C. C., Narayanan, D., Keres, D., et al. 2013b, MNRAS, 428, 2529

Hopkins, P. F., Younger, J. D., Hayward, C. C., Narayanan, D., \& Hernquist, L. 2010, MNRAS, 402, 1693

Husband, K., Bremer, M. N., Stanway, E. R., et al. 2013, MNRAS, 432, 2869 Kim, S., Stiavelli, M., Trenti, M., et al. 2009, ApJ, 695, 809

Klypin, A. A., Trujillo-Gomez, S., \& Primack, J. 2011, ApJ, 740, 102 Lagache, G., Cousin, M., \& Chatzikos, M. 2018, A\&A, 609, A130 Laporte, N., Pérez-Fournon, I., Calanog, J. A., et al. 2015, ApJ, 810, 130 Maiolino, R., Carniani, S., Fontana, A., et al. 2015, MNRAS, 452, 54 Marrone, D. P., Spilker, J. S., Hayward, C. C., et al. 2018, Natur, 553, 51 Mazzucchelli, C., Bañados, E., Decarli, R., et al. 2017, ApJ, 834, 83

McGreer, I. D., Fan, X., Strauss, M. A., et al. 2014, AJ, 148, 73

Mcmullin, J. P., Waters, B., Schiebel, D., Young, W., \& Golap, K. 2007, adass XVI, 376, 127

Miller, T. B., Chapman, S. C., Aravena, M., et al. 2018, Natur, 556, 469 Miller, T. B., Hayward, C. C., Chapman, S. C., \& Behroozi, P. S. 2015, MNRAS, 452, 878

Ota, K., Venemans, B. P., Taniguchi, Y., et al. 2018, ApJ, 856, 109

Planck-Collaboration, Ade, P. A. R., Aghanim, N., et al. 2014, A\&A, 571, A22 Popping, G., van Kampen, E., Decarli, R., et al. 2016, MNRAS, 461, 93 Riechers, D. A., Bradford, C. M., Clements, D. L., et al. 2013, Natur, 496, 329 Robson, E. I., Ivison, R. J., Smail, I., et al. 2014, ApJ, 793, 11 Stiavelli, M., Djorgovski, S. G., Pavlovsky, C., et al. 2005, ApJL, 622, L1 Strandet, M. L., Weiß, A., De Breuck, C., et al. 2017, ApJL, 842, L15 Swinbank, A. M., Karim, A., Smail, I., et al. 2012, MNRAS, 427, 1066 Trainor, R. F., \& Steidel, C. C. 2012, ApJ, 752, 39 Trakhtenbrot, B., Lira, P., Netzer, H., et al. 2017, ApJ, 836, 8 Utsumi, Y., Goto, T., Kashikawa, N., et al. 2010, ApJ, 721, 1680

Vallini, L., Gallerani, S., Ferrara, A., Pallottini, A., \& Yue, B. 2015, ApJ, 813,36

Wang, R., Wagg, J., Carilli, C. L., et al. 2013, ApJ, 773, 44 Willott, C. J., Bergeron, J., \& Omont, A. 2015a, ApJ, 801, 123 Willott, C. J., Carilli, C. L., Wagg, J., \& Wang, R. 2015b, ApJ, 807, 180 Willott, C. J., Omont, A., \& Bergeron, J. 2013, ApJ, 770, 13

Willott, C. J., Percival, W. J., McLure, R. J., et al. 2005, ApJ, 626, 657 Yamaguchi, Y., Kohno, K., Tamura, Y., et al. 2017, ApJ, 845, 108 Zheng, W., Overzier, R. A., Bouwens, R. J., et al. 2006, ApJ, 640, 574 